\title{
Representation of physical properties in the material properties open database
}

$\underline{\text { Edgar Eduardo Villalobos }}^{1}$, Luis E. Fuentes-Cobas ${ }^{1}$, Daniel Chateigner ${ }^{2}$, Giancarlo Pepponi ${ }^{3}$, Saulius Grazulis ${ }^{4}$, Veronica Barrera ${ }^{1}$, Rodrigo Dominguez ${ }^{1}$, Luis Fuentes-Montero 5

${ }^{1}$ Materials Physics Dept. Advanced Materials Research Center (CIMAV), Chihuahua, Mexico, ${ }^{2}$ Normandie Université, Université de Caen Normandie, CRISMAT-CNRS, Normandie, France, ${ }^{3}$ Center for Materials and Microsystems, Fondazione Bruno Kessler, Trento, Italy, ${ }^{4}$ Vilnius University, Faculty of Mathematics and Informatics, Vilnius, Lithuania, ${ }^{5}$ Diamond Light Source Ltd, Software Development Team, Didcot OX11 ODE, Oxfordshire, United Kingdom

E-mail: edgar.villalobos@cimav.edu.mx

The Material Properties Open Database (MPOD, http://mpod.cimav.edu.mx) is a functional element of the web-based open databases system linked with Crystallography. MPOD delivers single-crystal tensor properties in several representations, ranging from numerical matrices to 3D printing. Longitudinal moduli surfaces can be displayed in computers as well as in smart cell phones. Properties are stored as ".mpod" files. IUCr formatting standards (CIF) are followed. The original paper containing the data is cited. Structural and experimental information is also registered and linked. The MPOD system includes a physical properties dictionary with pertinent constitutive equations respecting Vol. D of the International Tables when possible. "Coupling properties", e.g. piezo-effects and magnetoelectricity, represent interactions linking different subsystems in a material. The implications of crystal symmetry in physical properties are systematically taken into account. Matrices' elements and longitudinal moduli surfaces are checked for consistency with the Neumann Principle. The representation of magnetic coupling properties and their link with magnetic symmetry concepts represent newly added features of MPOD. Color-symmetry and time-inversion considerations add complexity and interest to the task of systematizing the reception, validation and representation of this family of properties. The representation of polycrystals' properties constitutes a current challenge for the MPOD international group. Work on the systematization of the Voigt, Reuss and Hill approximations is described. The MPOD presentation includes a real-time demonstration of the database possibilities. Funding from Project CONACYT 257912 is acknowledged.

G. Pepponi, S. Grazulis, D. Chateigner: MPOD: A Material Property Open Database linked to structural information. Nuclear Instruments and Methods in Physics Research (2012), B284, 10-14.

L. Fuentes-Cobas, D. Chateigner, G. Pepponi et al: Implementing graphic outputs for the Material Properties Open Database (MPOD). Acta Cryst. (2014), A70, C1039.

Peter Moeck, Werner Kaminsky, Luis Fuentes-Cobas, Jean-Christophe Baloche, Daniel Chateigner: 3D printed models of materials tensor representations and the crystal morphology of alpha quartz. Symmetry, Culture and Science (2016), 27, 319-330.

\section{Magnetostriction - Terfenol-D}
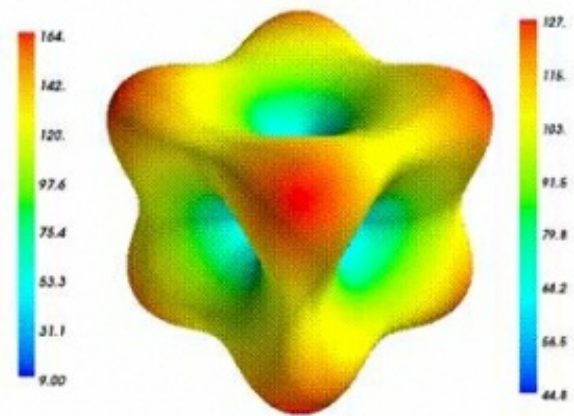

a) Single crystal

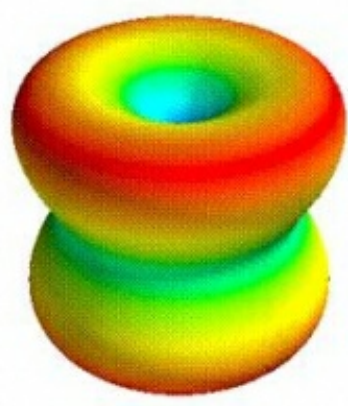

b) Axial texture

\section{Magnetoelectricity - $\mathrm{LiCoPO}_{4}$}

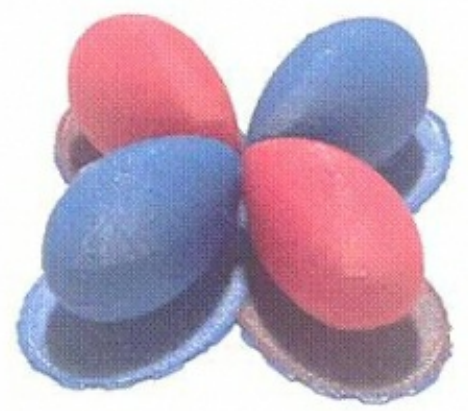

c) Single crystal, 3D print

Keywords: open database, properties, 3D printing 